\title{
Design and Characterization of a double-layered silicon charge detector for cosmic ray measurements in CALET
}

\author{
C. Avanzini, A. Basti, M.Y. Kim*, F. Morsani \\ INFN sez. di Pisa, V. Edificio C-Largo B. Pontecorvo 3, 56127 Pisa, Italy \\ E-mail: kim.meyoung@pi.infn.it
}

M. G. Bagliesi, K. Batkov, S. Bonechi, G. Bigongiari, R. Cecchi, P. Maestro, P. S. Marrocchesi, V. Millucci, R. Zei

University of Siena and INFN, V. Roma 56, 53100 Siena, Italy

\author{
O. Adriani, L. Bonechi, S. Bottai, G. Castellini, P. Papini, S. Ricciarini, \\ P. Spillantini, E. Vannuccini \\ University of Florence and INFN, V. Sansone 1, 50019 Sesto F.no, Florence, Italy
}

\begin{abstract}
CALET (CALorimetric Electron Telescope) is a space instrument to explore a new frontier at higher energies for the cosmic-rays (electrons, gamma rays and heavy nuclei) and to search for dark matter. ${ }^{1}$ This mission is designed for a long exposure observation on the external JEM-EF facility aboard the International Space Station. It is optimized for the search of nearby sources of acceleration of cosmic ray electrons in the $\mathrm{TeV}$ energy range and gamma rays up to several $\mathrm{TeV}$ range. It can also extend the available data on cosmic ray composition and on secondaryto-primary ratios to higher energies allowing to discriminate among different propagation models and to derive the acceleration spectra at the source. An accurate measurement of the charge of the incoming particle is performed in CALET by a double-layered array of pixelated silicon sensors (Silicon Array or SIA), covering a seamless sensitive area of the order of $0.3 \mathrm{~m}^{2}$ and providing single-element identification up to Fe and above. The design of SIA instrument and its characterization are presented.
\end{abstract}

9th International Conference on Large Scale Applications and Radiation Hardness of Semiconductor Detectors, RD09

September 30-October 2, 2009

Florence, Italy

\footnotetext{
* Speaker.
} 


\section{Introduction}

\subsection{CALET}

The CALET instrument consists of an imaging calorimeter (IMC), a total absorption calorimeter (TASC) and a silicon pad array (SIA) as in the Figure 1. The combination of IMC and TASC is designed to achieve high precision measurements of the cosmic ray (CR) electron spectrum in the range from $1 \mathrm{GeV}$ up to $10 \mathrm{TeV}$ and high energy resolution measurements of gamma rays in the range $20 \mathrm{MeV}$ to a few TeV. ${ }^{1}$ The combination of SIA and the calorimeter will allow to identify cosmic ray nuclei with individual element resolution and measure their energies up to 1000 $\mathrm{TeV}$ with an excellent energy resolution. CALET will be able to measure the absolute fluxes of individ-

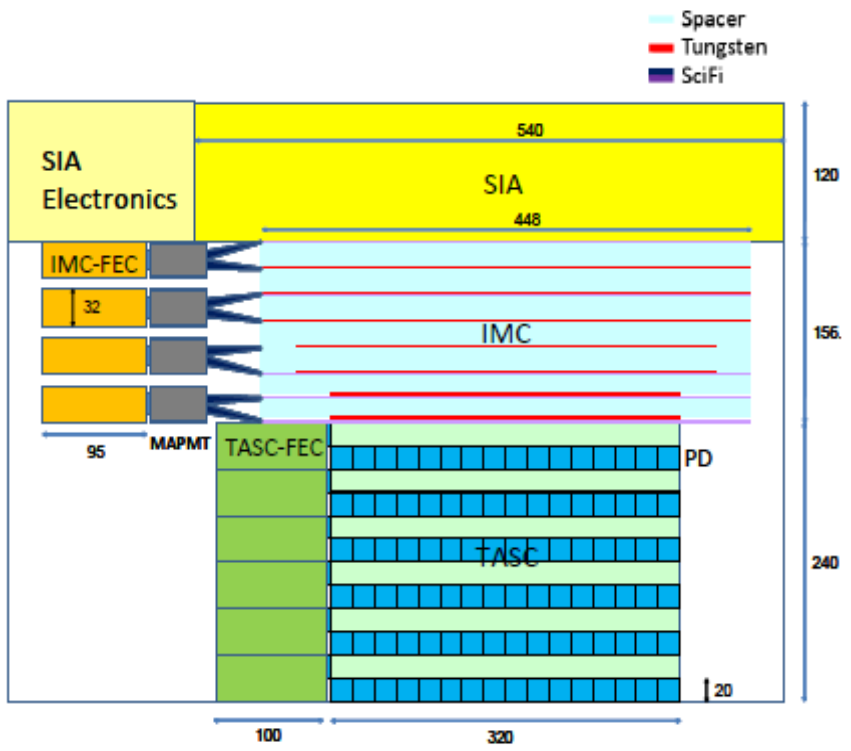

Figure 1: Scheme of CALET subdetectors ual elements and to extend to higher energies the present data on the secondary-to-primary ratios and cosmic ray composition from direct measurements and to verify the findings of balloon (ATIC, ${ }^{2}$ CREAM, ${ }^{3}$ TRACER $^{4}$ ) and space missions $\left(\mathrm{AMS}^{5}\right.$, PAMELA $\left.^{6}\right)$.

\subsection{Cosmic ray nuclei}

Cosmic ray nuclei in the energy range $100 \mathrm{MeV} / \mathrm{n}-100 \mathrm{GeV} / \mathrm{n}$ provide crucial information about the physical properties of the Galaxy. Some of them come unaltered from the sources (primaries), others (secondaries) are the result of the interaction of the primaries with the interstellar medium, or the disintegration of unstable species. Understanding the processes by which all these nuclei are produced, accelerated and propagated in the turbulent magnetic field of the Galaxy, will provide us an important information about the matter content and magneto-hydrodynamical properties of our Galaxy. It would even be possible to discover some evidence for new physics (e.g. SUSY dark matter) or astrophysical objects (e.g. primordial blackholes or stars made of antimatter). There are different propagation models with a strong dependence on the diffusion parameters. For their experimental determination, different classes of flux ratios are measured: primary-toprimary (e.g. C/O), secondary-to-primary (e.g. B/C or sub Fe/Fe), secondary-to-secondary(e.g. $\mathrm{Li} / \mathrm{B}$ or $\mathrm{Be} / \mathrm{B}){ }^{7}$ 


\section{Design and Characterisation of Silicon charge Detector}

\subsection{Silicon Sensor}

The baseline configuration of SIA is a mosaic of PIN diodes covering the active area without dead regions. A pixel geometry (pixel size of $11.25 \mathrm{~mm} \times 11.25 \mathrm{~mm}, 64$ pixels per sensor) has been chosen to isolate the ionization signal generated by the incoming particle, in the presence of albedo particles due to backscattering from the calorimeter. The pixel size was guided by the tradeoff between the impact point resolution from back extrapolation of the reconstructed track in the calorimeter and the total number of channels. The SIA detector consists of two layers of sensors which allow to have two independent measurements of the charge of the same particle with a great benefit in terms of background rejection and increase in the purity of the data sample when a single cosmic ray element is selected. The sensors are produced from 6 inch wafers of $500 \mu \mathrm{m}$ thickness with high resistivity $(>10 \mathrm{k} \Omega \cdot \mathrm{cm}$ ).
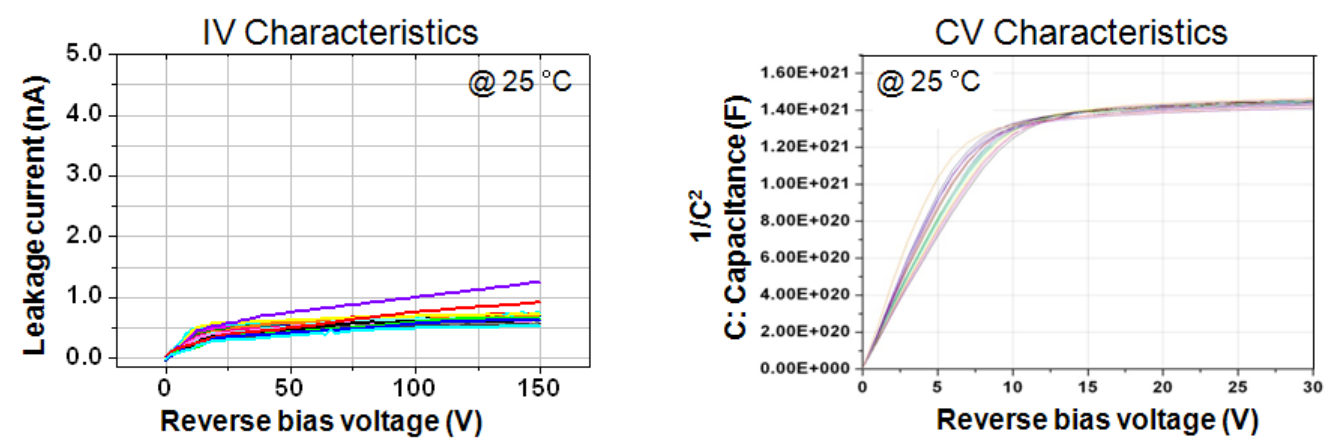

Figure 2: Electrical characteristics of silicon sensor

Figure 2 shows the electrical characteristics of 64 pixels on a sensor. The full depletion voltage is below $30 \mathrm{~V}$. The dark current per pixel typically lower than $1 \mathrm{nA}$ and the capacitance per pixel is $25 \mathrm{pF}$ at the full depletion.
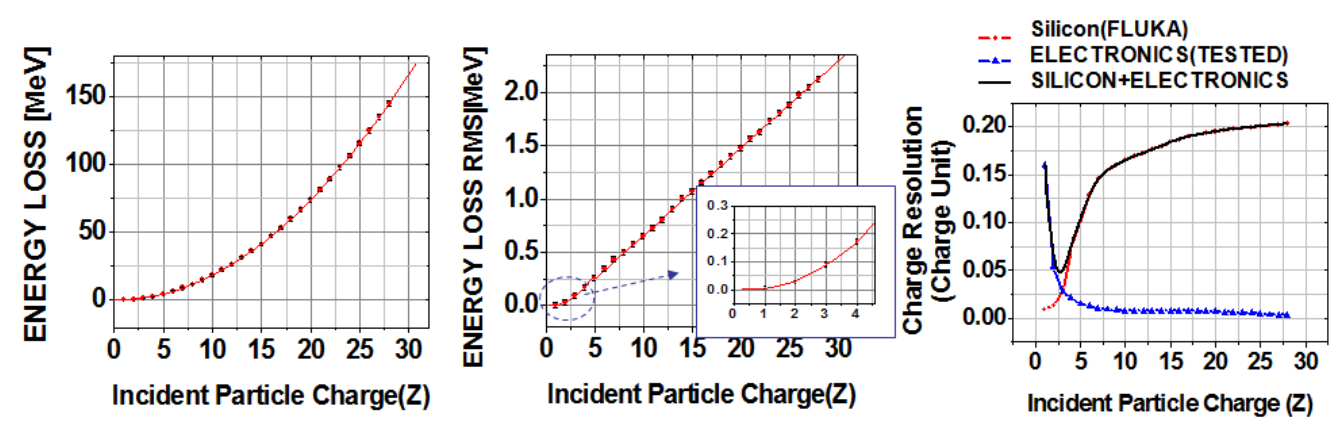

Figure 3: Energy loss in $500 \mu \mathrm{m}$ Thick silicon FLUKA simulation.

An important requirement for a direct measurement of $\mathrm{CR}$ composition is the ability to identify individual chemical elements in the cosmic ray flux. The $Z^{2}$ dependence of the specific ionization loss of an ultrarelativistic nucleus of charge $\mathrm{Z}$ in a thin silicon sensor can provide a sufficient charge discrimination capability, provided the electronics noise is kept sufficiently low. The energy loss 
(average and variance) for $10 \mathrm{GeV} / \mathrm{n}$ nuclei of charge $\mathrm{Z}=1$ to 28 are shown in Figure 3. The third graph on Figure 3 shows the charge resolution which is defined as the ratio between the rms and the average energy loss expressed in terms of the unitary elementary charge. The simulated charge resolution up to the Iron group is within 0.2 charge unit which is limited by the intrinsic energy straggling in the silicon sensor.

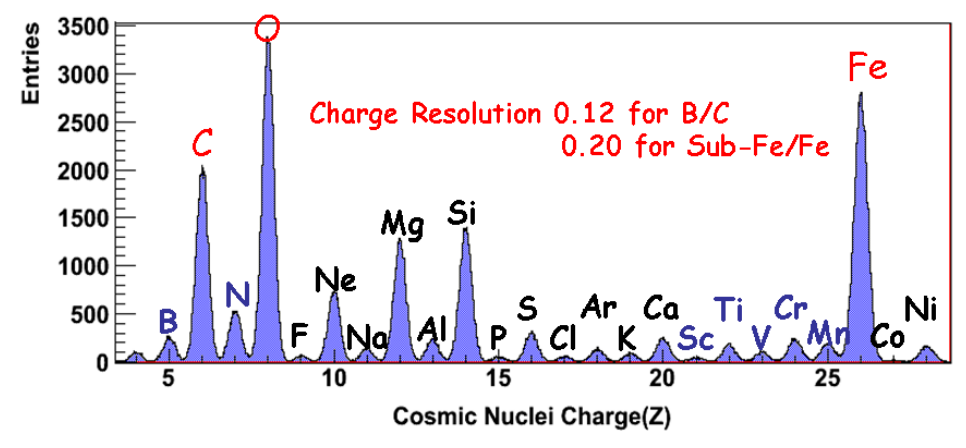

Figure 4: Expected abundances of cosmic ray

Figure 4 shows the expected cosmic ray abundances from SIA. In this simulation, the relative abundance of each chemical element is taken from data on the charge composition of the HEAO- ${ }^{8}$ experiment at $\mathrm{E}=16 \mathrm{GeV} / \mathrm{n}$ and from the $\mathrm{SOKOL}^{9}$ experiment at $\mathrm{E}=2.5 \mathrm{TeV}$.

\subsection{The readout architecture}

Each pair of sensors (128 pixels) is readout by a dedicated board (VAB) hosting 4 chips of the VA family. The front-end chip VA32-HDR14.2 (a sample-and-hold, low-noise, low-power, large dynamic range ASIC with multiplexed readout of 32 channels) with epitaxial layer protection against SEE effects was developed under the support of INFN. ${ }^{10}$ The chip is optimized for positive charge inputs. The VAB board implements a sequencer for the readout of 4 VA chips with 16 bits digitization using 4 independent ADC integrated circuits. Figure 5 shows the gain curve and the linearity of the VAB board. It allows us to measure the cosmic nuclei up to $\mathrm{Z}=33$ with a good linearity.
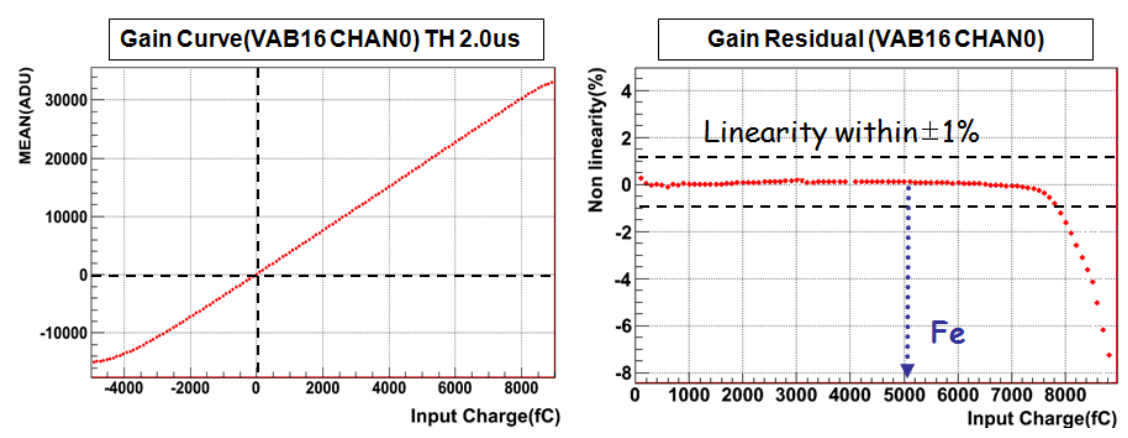

Figure 5: Characteristics of the front end electronics

The 6 VAB boards of each ladder of the SIA are readout by a Ladder Controller (LAC) that formats the sub-event. The LAC also supervises the distribution of trigger signals. Gain calibrations 
of individual channels are performed by each VAB under ther control of the LAC by using a charge injection facility built into the ASIC. The global readout and sparsification of the event fragments from the LAC boards is done by a ReadOutController (ROC) interfaced to the main DAQ.

\subsection{Mechanical design}
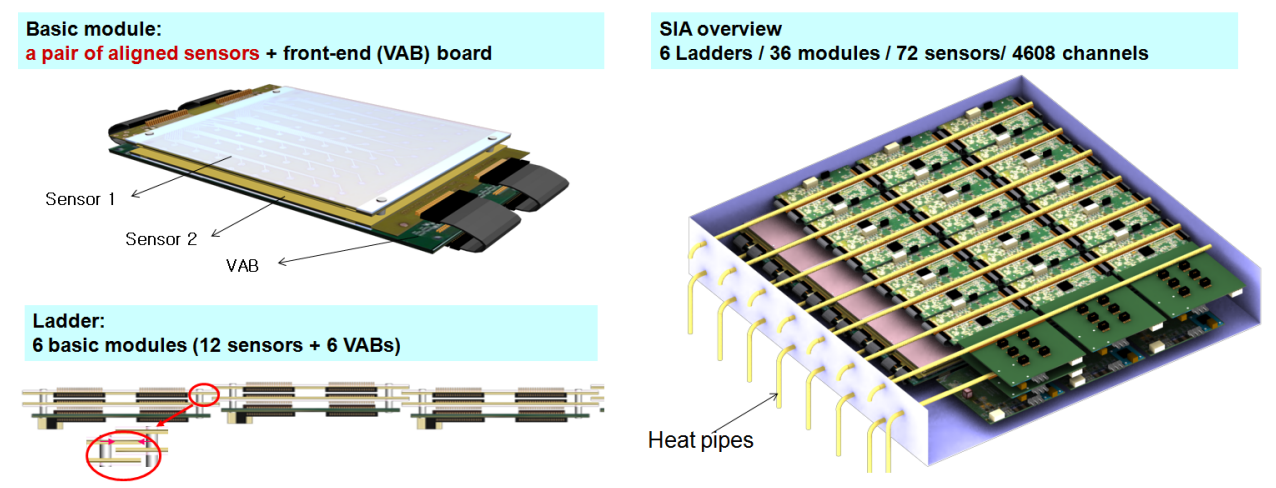

Figure 6: System mechanical design

The SIA detector consists of two layers of sensors for a total of 72 units. The basic unit consists of sensors connected to a VAB board (Left uppder on Figure 6). Along each ladder, 6 modules are arranged to allow for the overlapping of the sensors(Left lower on Figure 6). The SIA is mechanically divided in two halves: a lower section where 3 (odd numbered) ladders are arranged and an upper section that provides the mechanical support for the remaning 3 (even numbered) ladders(Right on Figure 6). The design allows to achieve a complete overlap of all sensors, while providing two independent measurements of the charge of the incoming cosmic ray within the acceptance of CALET. The readout of the two layers is integrated into the mechanical structure. All the electronics cards are placed on the upper and lower surfaces of the detector in a symmetric way. This simplifies the design of the cooling system ((based on the use of heat pipes) and minimizes temperature gradients across the instrument.

\section{Detector test with Atmospheric Muons}

\subsection{Muon test setup}
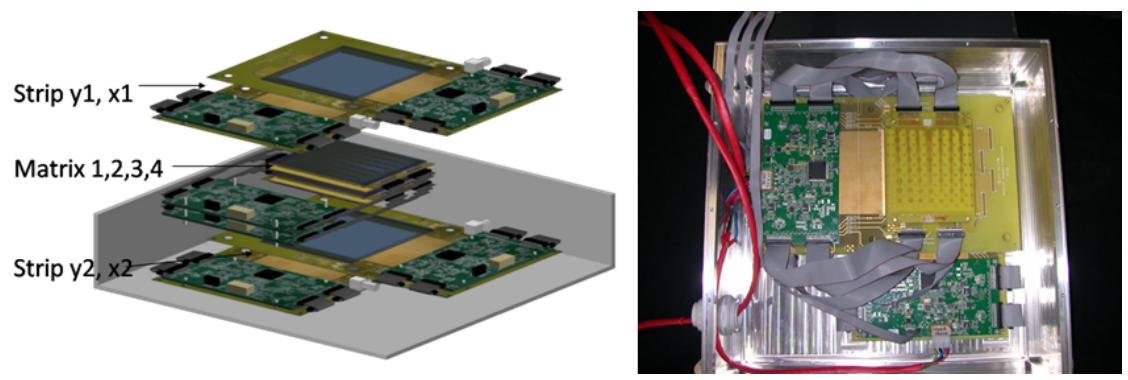

Figure 7: Beam tracker 
A beam tracker using the basic modules of SIA for CALET was built for the beamtest 2009 in CERN (Figure 7). Four layers of matrix sensors are aligned to each other and also 4 strip sensors (positioned to $\mathrm{x}$ and $\mathrm{y}$ ) are added for the tracking. A lab test was performed where the large area silicon sensors were triggered by atmospheric muons using an external coincidence of 3 scintillators.

\subsection{Muon test result}
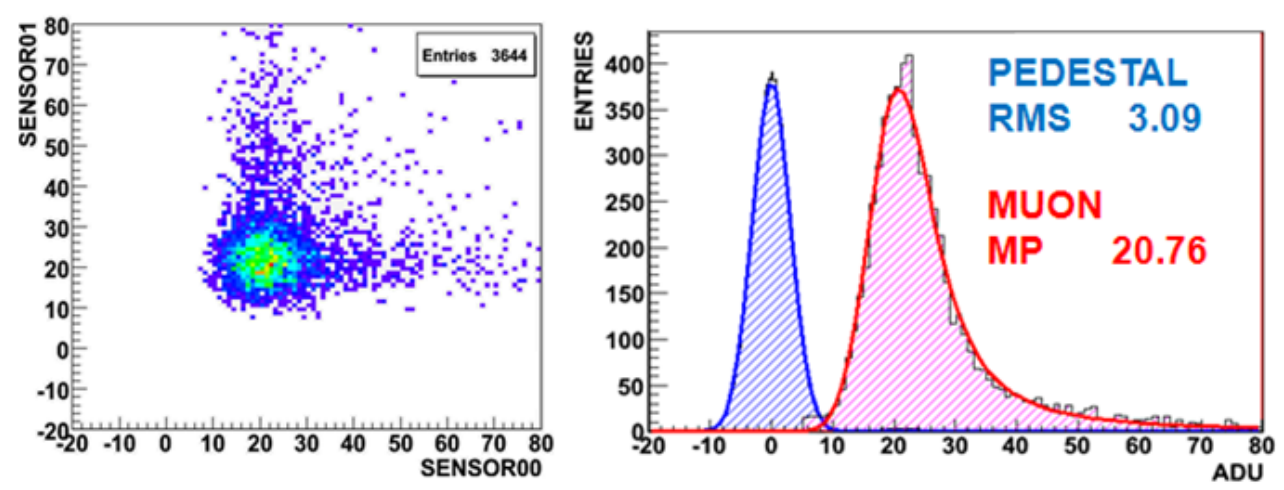

Figure 8: Atmospheric Muon test (L: Correlation plot, R:Signal to Noise)

To calculate the $\mathrm{S} / \mathrm{N}$ of the system, we select vertically incident events using the information of 4 sensors only if the position of the fired pixels are correlated. About 3600 events are analyzed where on average 25 pixels were illuminated due to the trigger geometry. The pulse height correlation of the selected event is shown in Figure 8. We first fit the rms of the inclusive pedestal distribution from 25 pixels. Then, the muon signal is fitted with a Landau distribution convoluted with a Gaussian. As a result, the most probable value of the signal-to-noise ratio of the system is about 7 .

\begin{tabular}{|l|l|}
\hline Sensor & Efficiency (\%) \\
\hline Sensor 00 & $99.90 \pm 0.06$ \\
Sensor 01 & $99.53 \pm 0.13$ \\
Sensor 02 & $99.03 \pm 0.17$ \\
Sensor 03 & $99.43 \pm 0.14$ \\
\hline
\end{tabular}

Table 1: Detection efficiency

The detection efficiency is calculated when 3 other sensors have a pulse height larger than 5 sigma of the pedestal. Table 1 shows the average efficiency of the matrix sensor is about $99.5 \%$. A more accurate determination of the efficiency was performed with a larger statistics from the analysis of the data collected at a beam test that took place in August 2009 at the SPS of CERN. The results, fully consistent with the above, will be reported in a dedicated paper.

\section{Plan and Conclusion}

CALET has successfully completed the Phase-A mission studies and it is scheduled for a launch to the International Space Station (ISS) at the end of 2013 by the Japanese Transfer Vehicle (HTV). The instrument has been designed to perform science observations for at least 5 years on the External Facility of the Japanese module (JEM-EF) on the ISS. The results of the SIA tests, confirm that the design parameters are compliant with the science requirements of the mission. The 
production and assembly of the SIA and its environmental tests are foreseen to take place in the time frame of about 2 years, followed by the integration of the instrument in the payload.

\section{References}

[1] S. Torii et al. Nucl.Phys.B (Proc.Suppl.) 166 (2007) 43-49

[2] A. D. Panov et al. Advances in Space Research 37 (2006) 1944-1949

[3] E. S. Seo et al. Advances in Space Research 33 (2004) 1777-1785

[4] A. Oliva et al. Nucl. Phys.A 588 (2008) 255âĂŞ258

[5] P. Picozza et al. 30th ICRC, Vol. 2 (OG part 1), 19âĂŞ22

[6] D. Muller et al. 30th ICRC, Merida, 2007

[7] D. Maurin et al. arXiv:astro-ph/0212111

[8] Engelmann et al. Astron.Astrophys. Vol.233 (1990) 96

[9] Ivanenko e tal. 23th ICRC, Calgary, Vol. 2(1993), 17

[10] M.G.Bagliesi et al. Nucl.Phys.B (Proc.Suppl.) 172 (2007) 156-158 